\title{
Engineered Solder-Directed Self-Assembly Across Length Scales
}

Robert Knuesel, Shameek Bose, Wei Zheng, and Heiko O. Jacobs

Department of Electrical and Computer Engineering, University of Minnesota, 200 Union Street SE, Minneapolis, MN, 55455

\begin{abstract}
We report on recent progress in the directed self-assembly of discrete inorganic semiconductor device components. Different from prior research, the goal is to enable the integration of increasingly small dies while supporting unique-angle orientation and contact pad registration. The process is based on the reduction of surface free energy between liquid solder coated areas on the substrate and metal-coated binding sites on the semiconductor dies. Recent advances include flip-chip assembly with unique angular orientation accomplished using "twoelement" docking sites that contain pedestals that act as chaperones for the solder directed assembly to take place. The scale reduction to $20 \mu \mathrm{m}$ sized components involves the use of a liquid-liquid interface to concentrate component delivery and speed up the self-assembly process to prevent oxidative dissolution of the solder sites prior to completion.
\end{abstract}

\section{INTRODUCTION}

The construction of man-made artifacts such as cell phones and computers relies on robotic assembly lines that place, package, and interconnect a variety of devices that have macroscopic $(>1 \mathrm{~mm})$ dimensions ${ }^{[1]}$. The key to the realization of these systems is our ability to integrate/assemble components in $2 \mathrm{D} / 3 \mathrm{D}$ as well as link/interconnect the components to transport materials, energy, and information. The majority of these systems that are on the market today are heterogeneous in nature. Heterogeneous systems can be characterized as systems that contain at least two separate parts, thereby prohibiting monolithic integration. Such systems are typically fabricated using robotic pick and place. The size of the existing systems could be reduced by orders of magnitudes if microscopic building blocks could be assembled and interconnected effectively ${ }^{[2]}$. The difficulty is not the fabrication of smaller parts, but their assembly into an interconnected system. For components with dimensions less than $100 \mu \mathrm{m}$, adhesive capillary forces often dominate gravitational forces, making it difficult to release the components from a robotic manipulator ${ }^{[3]}$. As a direct result, heterogeneous integration using an extension of serial robotic pick and place and wire-bonding has not proven a viable solution.

At the other extreme, nature forms materials, structures, and living systems by selfassembly on a molecular length scale ${ }^{[4,5]}$. As a result, self-assembly based fabrication strategies are widely recognized as inevitable tools in nanotechnology and an increasing number of studies are being carried out to "scale-up" these concepts to close the assembly gap between nanoscopic and macroscopic systems. Recent demonstrations of processes that can assemble micrometer to millimeter-sized components include: shape-directed fluidic methods that 
assemble trapezoidal parts on planar surfaces on the basis of gravity and complementary 3D shapes ${ }^{[6,7]}$, liquid-solder-based self-assembly that uses the surface tension between pairs of molten solder drops to assemble functional systems ${ }^{[8-10]}$, capillary force-directed self-assembly that uses hydrophilic/hydrophobic surface patterns and photo-curable polymers to integrate micro-optical components, micro-mirrors and semiconductor chips on silicon substrates ${ }^{[11-13]}$, and sequential shape-and-solder-directed self-assembly that combines 3D shapes to define a chaperone with solder directed assembly to effectively assemble electrically interconnected parts [14-19]. The sequential shape-and-solder-directed self-assembly process has been applied to flipchip assembly with unique contact pad registration ${ }^{[16]}$ as well as the packaging of light emitting diodes ${ }^{[14-16]}$ and transponders that can be interrogated remotely ${ }^{[17]}$. Comparing more recent concepts ${ }^{[18,19]}$ with the pioneering work by Yeh and Smith ${ }^{[6,7]}$, there are a number of fundamental differences: Recent methods do not require trapezoidal chips to prevent upside down assembly and asymmetric L or T shaped chip designs for angular orientation control. Instead they use simple shapes or openings in combination with solder coated areas to enable assembly in 3D or in 2D considering flip-chip assembly with unique angular orientation and contact pad registration. The openings are bigger than the components and act as guides for the solder directed self-assembly process. The solder drives the assembly into the aligned stable position and the driving force is surface tension, as opposed to gravity. Recent studies have also overcome the difficulty in assembling more than one component type through sequential methods that either use activation of selected receptors ${ }^{[20]}$ or different sized openings ${ }^{[14-16]}$. Unique orientation no longer requires asymmetric three dimensional chips including triangular, $\mathrm{L}$ or $\mathrm{T}$ shaped parts that are not very common. While a number of applications have been demonstrated, scalability to smaller dimensions remains a challenge. Stauth et al. has successfully tested shape and solder directed self-assembly with $100 \mu \mathrm{m}$ sized components ${ }^{\text {[21], }}$ which are three times smaller than our previously reported parts ${ }^{[16]}$. This paper extends prior work ${ }^{[10,14-16]}$ in the area of shape and solder directed self-assembly to smaller scales. It reports on angular orientation control, flip-chip assembly, and recent progress in the assembly of ultra small chiplets with a side length of $20 \mu \mathrm{m}$. A new system employing a liquid-liquid interface will be presented to deliver and concentrate components with correct pre-orientation.

Figure 1

Self-assembly of dies using A) solder-based alignment alone, B) wells for size discrimination, and C) combined pedestals -, solder -, and IO layouts that supports single angular orientation and flip-chip contact pad registration.

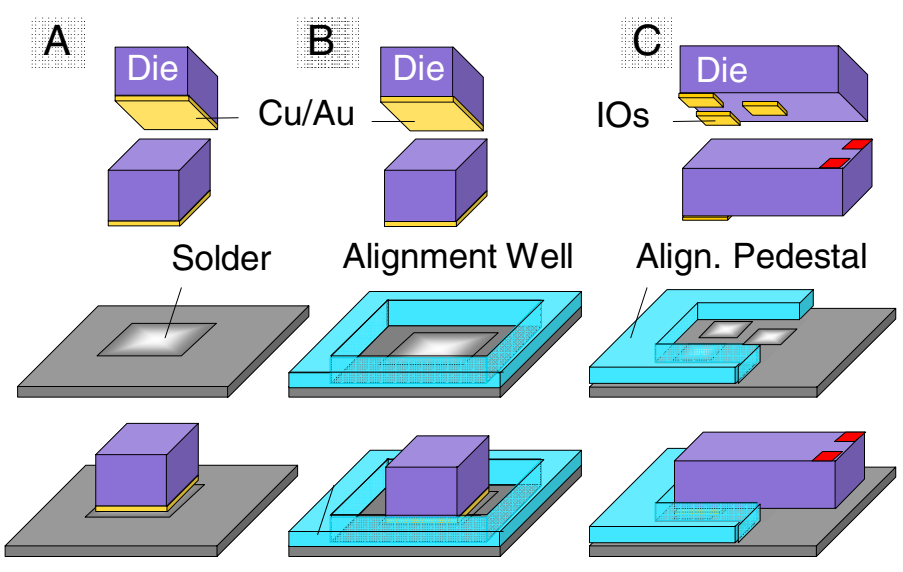

Figure 1 illustrates the basic concept of solder directed self-assembly introducing alignment pedestals and contact pad layouts with increasing complexity. The layout B prevents assembly of more than one component type onto a single receptor, while the layout in $\mathrm{C}$ enables 
flip-chip assembly with single angular orientation and contact pad registration forming multiple contacts to the device; components can only attach to the solder-coated areas if a correct angular pre-orientation condition is met: components that arrive at the docking sites with an angular orientation that deviates by more than $\pm 90^{\circ}$ from the desired orientation will not find a sufficient overlap between the binding site (contact area) on the components and the solder-coated areas and will not attach. Other components will be captured and aligned due to the reduction of the interfacial free energy.

\section{EXPERIMENT}

Figure 2 shows the sequential self-assembly procedure that we employ to batch-assemble different sized components with a single-angle orientation. The assembly was performed in a glass vial that was filled with ethylene glycol at a temperature of $150{ }^{\circ} \mathrm{C}$ so the solder was molten. Ethylene glycol was used to accommodate the higher melting point solder that is not compatible with a water-based assembly solution. We used both a low- $\left(47^{\circ} \mathrm{C}\right)$ and medium$\left(138{ }^{\circ} \mathrm{C}\right.$ ) melting-point (mp) solders (Y-LMA-117 and LMA-281, Small Parts, Miami Lakes, FL) in our experiments; we did not observe a notable difference between the two. The ethylene glycol solution was made slightly acidic $(\mathrm{pH} \sim 4.0)$ with hydrochloric acid to remove metal oxide from the surface of the solder drop; an oxide layer that - if sufficiently thick — blocked the wetting of the metal surface. Component transport and mixing was provided by hand agitation of the vial/drum to accomplish a tumbling motion across the surface, a motion that needs to be automated in future work.

\section{Figure 2}

Rotational agitation procedure showing a two-step process to integrate multiple types of chiplets on a single substrate.

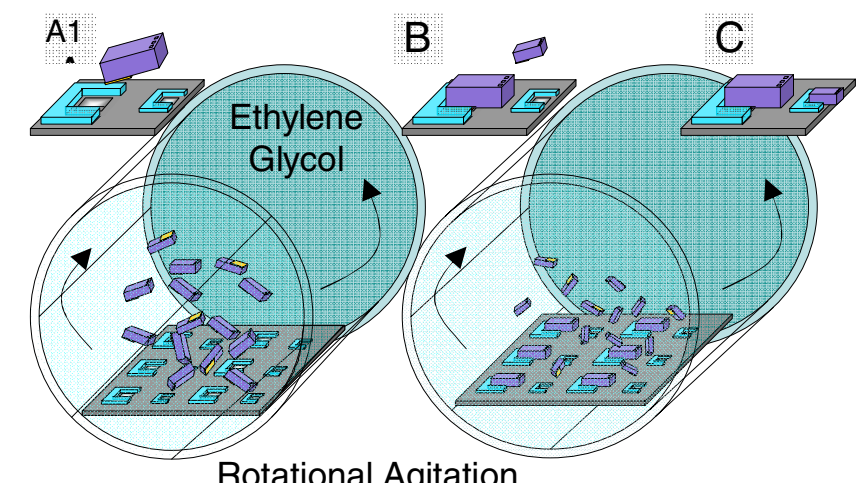

Rotational Agitation

Through the course of this study we tested a number of different components including GaAlAs-LEDs, Si, glass, and SU-8 blocks with different pad layouts. Figure 3 represents a summary of the results. Figure 3A illustrates 1500 silicon chiplets, $300 \mu \mathrm{m}$ on a side, which assembled onto a flexible polyimide surface with $98 \%$ coverage. With $~ 5000$ components inside the vials, the assembly took about $90 \mathrm{~s}$ to reach steady state and was completed in 3 minutes. The lateral and angular precision was $\sim 15 \mu \mathrm{m}$ and $\sim 3^{\circ}$, respectively, and limited by non-uniformity of the components that were fabricated by dicing using a dicing saw. Figure $3 \mathrm{~B}$ shows GaAs/GaAlAs light-emitting diodes that have been assembled on a silicon substrate. The insets show the LEDs under operation. A potential problem that we have observed is highlighted in the inset (Figure 5A). Without alignment pedestals, two chiplets can occupy a single receptor which is considered a defect. This defect can be eliminated using alignment pedestals as illustrated in Figure 3C. The entire assembly contained 196 interconnected LEDs with a side length of 280 
$\mu \mathrm{m}$. The chiplets assembled with four stable orientations $0^{\circ}, 90^{\circ}, 180^{\circ}$, and $270^{\circ}$. Figure $3 \mathrm{E}$ is an example of sequential batch transfer showing a 10×10 array that contains $900 \mu \mathrm{m}$ and $500 \mu \mathrm{m}$ sized dies assembled using a two-step self-assembly sequence. The largest components were $2 \times 1 \times 1 \mathrm{~mm}$ glass blocks. The blocks assembled with unique orientation and attached to 7 contacts on the substrate that are visible from the top. The smallest components were $280 \mu \mathrm{m}$ on a side (Figure 3C).

\section{DISCUSSION}

Solder directed self-assembly can be scaled down to much smaller dimensions than what has been demonstrated. The surface free energy of liquid solder dominates thermal energy and Brownian motion down to the sub-1 $\mathrm{mm}$ scale. In practice, however, scaling has been challenging. The problem is a surface oxide that forms due to residual oxygen, blocking the selfassembly. To combat this problem, small amounts of acid are added ( $\mathrm{pH} \mathrm{2-4)} \mathrm{to} \mathrm{the} \mathrm{assembly}$ solution. The acid, however, leads to an oxidative dissolution of the solder an issue which becomes increasingly challenging as the solder volume is reduced. There are a number of potential solutions to this problem. In this study we present a new component delivery mechanism to speed up the self-assembly process by concentrating the number of available components. The process is illustrated in Figure 4.

Figure 3

Summary of selfassembly results using GaAlAsLEDs, Si, Glass, and SU-8 blocks with varying docking site layouts including solder-directed assembly of A) silicon parts and B) LEDs, C) well assembly, D) single-angle orientation assembly using "two-step" docking sites, E) contact pad, two step registration, and F) flip-chip assembly of parts with multiple IO connections.

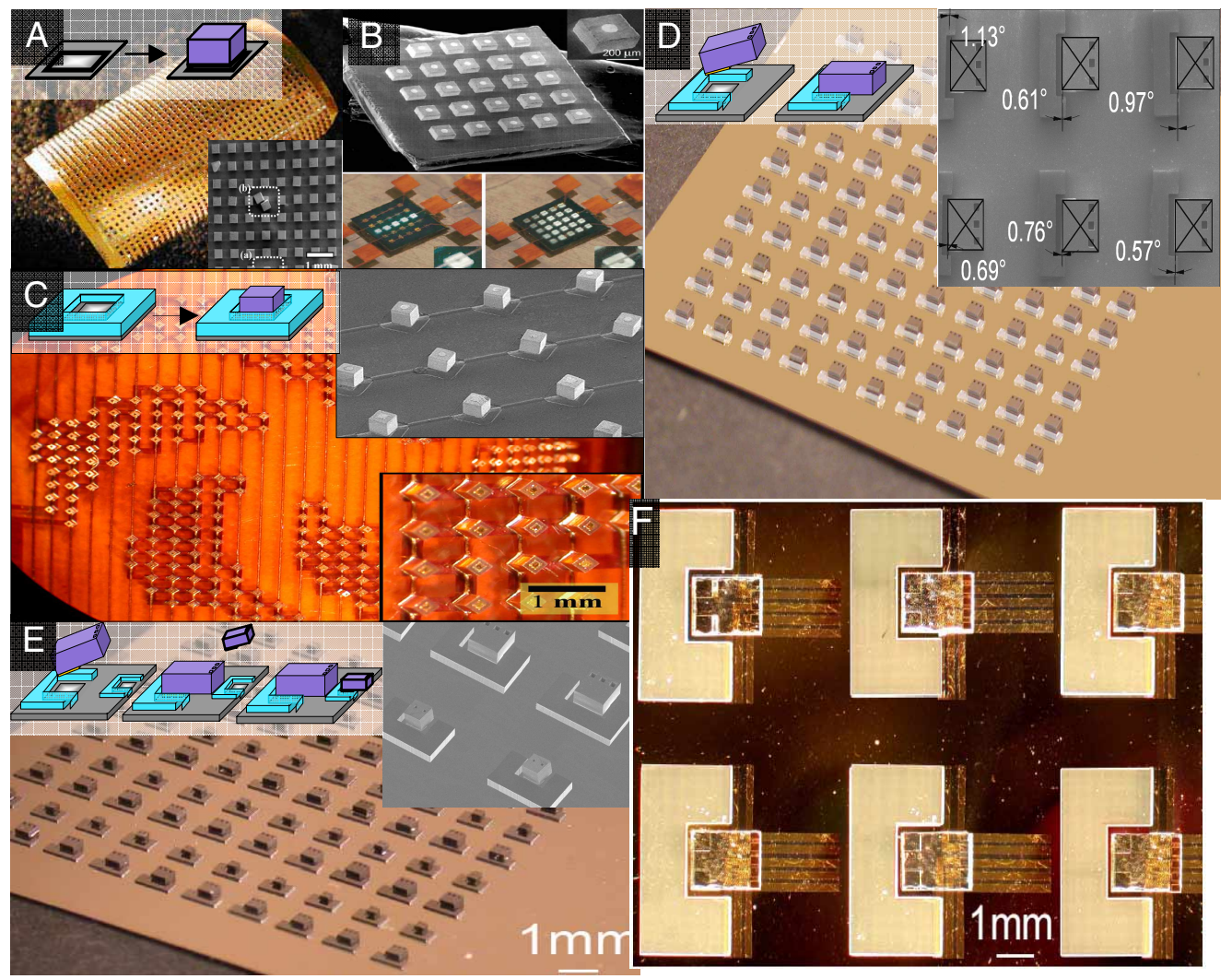

The components are injected into the silicon oil and settle at the oil/water interface. We tested SU-8 and Si blocks that were formed through etching carrying a single gold contact on one side. The components were plasma treated in oxygen plasma prior to use and settled at the oil water interface with the gold surface facing the oil phase and the remainder facing the water. 
There has been a substantial body of work on self-assembly at the liquid-liquid interface that used the static wetting angle between components. The illustrated concept does not depend on or use the static wetting angle. Instead it makes use of the dynamic angle for a given liquid - liquid solid interface. The sheer velocity and viscosities determine the shape of the interface. Both up and downward slopes are possible. When the substrate is immersed into the liquid, the angle changes dramatically and components are delivered and concentrated in a thin surface layer. Downward motion results in rapidly accelerated self-assembly. The results show $20 \mu \mathrm{m} \mathrm{SU-8}$ components that self-assembled after dipping the chiplets $\sim 10$ times into the solution. Withdrawing the sample reverses the angle and effectively removes unassembled components.
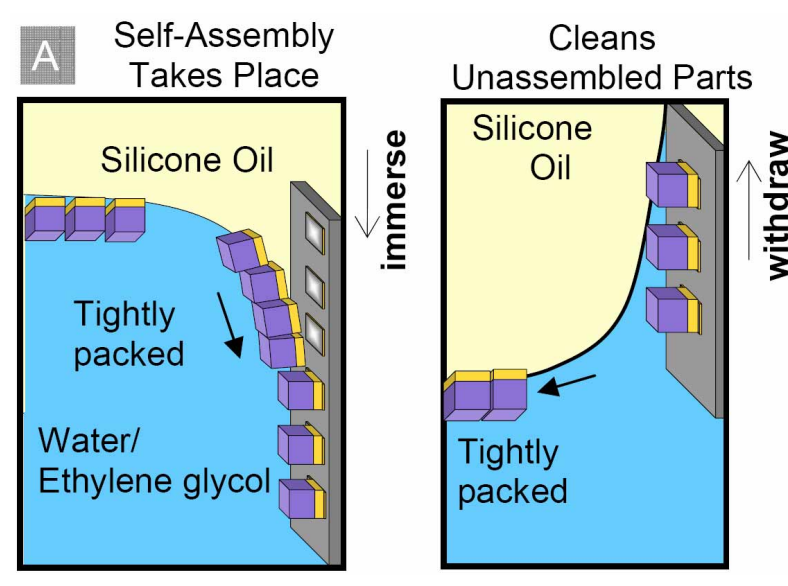

Figure 4

Self-assembly of ultra-small components using a liquid-liquid interface to increase the concentration of components that are available for assembly. A) Diagram showing the substrate being pushed through a silicone oil - water interface.

B) Optical and C,D) scanning electron micrograph images of assembled components.

While the concept works well, we observed some level of defects. Figure 5 shows defects that have been observed such as two components on a single receptor and misaligned components due to an imperfect solder/metal coating. Similar to our previous studies ${ }^{[14-19]}$, we expect that these defects can be eliminated through proper engineering of the process.

\section{Figure 5}

Observed defects resulting from ultra-small fluidic self assembly: A) More than one component per receptor site, and B) flawed orientation.

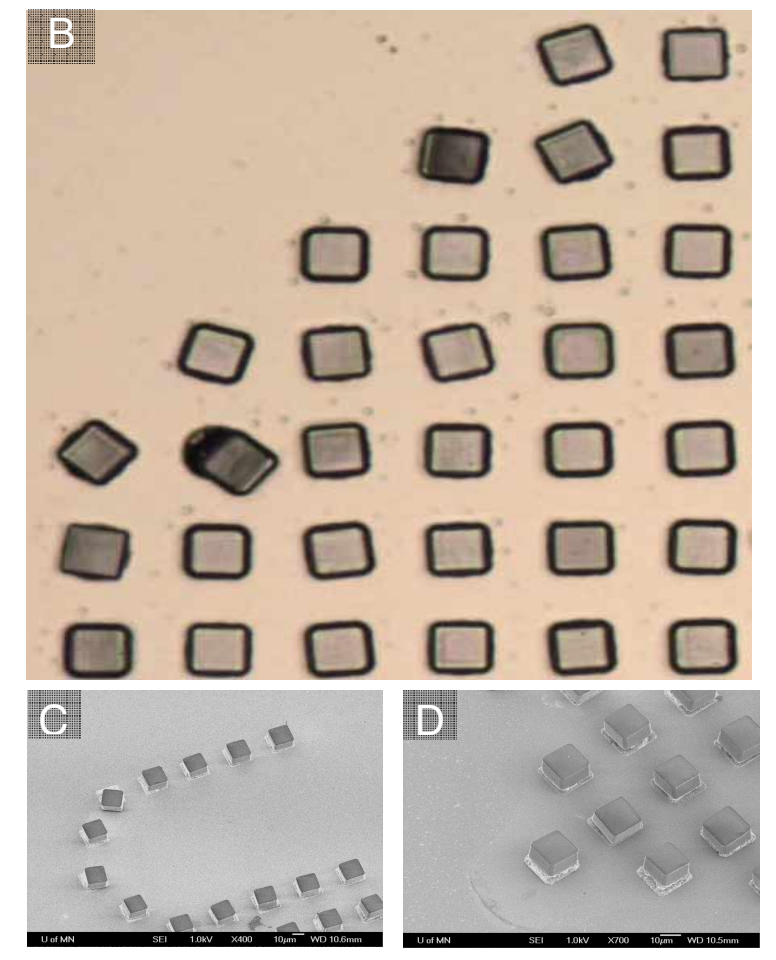




\section{CONCLUSIONS}

In summary, we have demonstrated the directed self-assembly of micrometer-sized components with single-angular orientation accuracy of $0.3^{\circ}$ and contact-pad registration for $280 \mu \mathrm{m}-2 \mathrm{~mm}$ sized dies. Alignment pedestals and registration to 7 contact pads has been shown providing a route to flip-chip self-assembly. Scaling down to 10-fold smaller chip dimensions, however, was not possible by a linear extension of the previous agitation concepts. Instead a new concept has been developed that makes use of a dynamic method that immerses the chip through a liquid-liquid interface. While the feasibility has been tested with $20 \mu \mathrm{m}$ sized dies, we have not yet established the ultimate complexity in terms of alignment accuracy, number of contacts, and density of interconnects that could be established by this process. A rough estimate of the alignment accuracy for the smallest dies is $1-2 \mu \mathrm{m}$.

\section{REFERENCES}

[1] M. B. Cohn, K. F. Bohringer, J. M. Noworolski, A. Singh, C. G. Keller, K. Y. Goldberg, R. T. Howe, Proceedings of SPIE 1998, 3512, 2.

[2] T. D. Clark, J. Tien, D. C. Duffy, K. E. Paul, G. M. Whitesides, Journal of the American Chemical Society 2001, 123, 7677.

[3] R. S. Fearing, Proceedings 1995, 212.

[4] S. Zhang, Nature Biotechnology 2003, 21, 1171.

[5] G. M. Whitesides, B. Grzybowski, Science 2002, 295, 2418.

[6] H. J. J. Yeh, J. S. Smith, IEEE Photonics Technology Letters 1994, 6, 706.

[7] J. S. Smith, H. J. J. Yeh, US Patent 1998, 5,824,186.

[8] D. H. Gracias, J. Tien, T. L. Breen, C. Hsu, E. M. Whitesides, Science 2000, 289, 1170.

[9] M. Boncheva, D. H. Gracias, H. O. Jacobs, G. M. Whitesides, Proc. Natl. Acad. Sci. USA 2002, 99, 4937.

[10] H. O. Jacobs, A. R. Tao, A. Schwartz, D. H. Gracias, G. M. Whitesides, Science 2002, 296, 323.

[11] U. Srinivasan, D. Liepmann, R. T. Howe, Journal of Microelectromechanical Systems 2001, 10, 17 .

[12] U. Srinivasan, M. A. Helmbrecht, C. Rembe, R. S. Muller, R. T. Howe, IEEE Journal of Selected Topics in Quantum Electronics 2002, 8, 4.

[13] K. F. Böhringer, U. Srinivasan, R. T. Howe, Interlaken, Switzerland, 2001.

[14] W. Zheng, P. Buhlmann, H. O. Jacobs, Proc. Natl. Acad. Sci. USA 2004, 101, 12814.

[15] W. Zheng, H. O. Jacobs, Applied Physics Letters 2004, 85, 3635.

[16] W. Zheng, H. O. Jacobs, Advanced Functional Materials 2005, 15, 732.

[17] F. Patolsky, G. Zheng, C. M. Lieber, Analytical Chemistry 2006, 78, 4260.

[18] H. O. Jacobs, Z. Wei, USA, Provisional Application, March 2006.

[19] C. R. Barry, H. O. Jacobs, Nano Letters 2006, 6, 2790.

[20] X. Xiong, Y. Hanein, J. Fang, Y. Wang, W. Wang, D. T. Schwartz, K. F. Bohringer, Journal of Microelectromechanical Systems 2003, 12, 117.

[21] S. A. Stauth, B. A. Parviz, Proceedings of the National Academy of Sciences of the United States of America 2006, 103, 13922. 